\title{
24-Hour Glucose Profiles during Continuous or Oscillatory Insulin Infusion Demonstration of the Functional Significance of Ultradian Insulin Oscillations
}

\author{
Jeppe Sturis, André J. Scheen, * Rachel Leproult, Kenneth S. Polonsky, and Eve Van Cauter \\ Department of Medicine, University of Chicago, Chicago, Illinois 60637; and *Division of Diabetes, Nutrition and Metabolic Diseases, \\ Department of Medicine, Université de Liège, B-4000 Belgium
}

\begin{abstract}
Under basal and stimulated conditions, normal insulin secretion oscillates with periods in the ultradian 100-150-min range. To test the hypothesis that oscillatory insulin delivery is more efficient in reducing blood glucose levels than continuous administration, nine normal young men were each studied on two occasions during a 28 -h period including a period of polygraphically recorded sleep. Endogenous insulin secretion was suppressed by somatostatin, a constant intravenous glucose infusion was administered, and exogenous insulin was infused either at a constant rate or in a sinusoidal pattern with a period of $120 \mathrm{~min}$. The mean glucose level over the $28-\mathrm{h}$ period was $0.72 \pm 0.31 \mathrm{mmol} / \mathrm{liter}$ lower when insulin was infused in an oscillatory pattern than when the rate of infusion was constant $(P<0.05)$. The greater hypoglycemic effect of oscillatory versus constant infusion was particularly marked during the daytime, with the difference averaging $1.04 \pm 0.38 \mathrm{mmol} /$ liter $(P<0.03)$. Serum insulin levels tended to be lower during oscillatory than constant infusion, although the same amount of exogenous insulin was administered under both conditions. Ultradian insulin oscillations appear to promote more efficient glucose utilization. (J. Clin. Invest. 1995. 95:1464-1471.) Key words: glucose utilization $\cdot$ receptor binding $\bullet$ pulsatility • diurnal variations $\cdot$ diabetes
\end{abstract}

\section{Introduction}

Secretion in a pulsatile or oscillatory pattern rather than at a constant rate seems to be the rule in endocrine systems. The physiological significance of pulsatile hormone secretion was first demonstrated when it was shown that intermittent, rather than continuous, delivery of GnRH was essential for normal reproductive function (1). Physiologic implications of pulsatile release with frequencies in the so-called "ultradian" range (i.e., 1-3 h) have been since demonstrated for most hormones of the hypothalamo-pituitary axis (2).

Oscillatory activity in the ultradian range is also prominent in the temporal organization of beta-cell secretion. Ultradian oscillations in glucose concentrations and insulin secretion have

Address correspondence to Eve Van Cauter, Ph.D., Department of Medicine, MC 1027, University of Chicago, 5841 South Maryland Avenue, Chicago, IL 60637. Phone: 312-702-0169; FAX: 312-702-9194.

Received for publication 6 September 1994 and in revised form 14 December 1994.

J. Clin. Invest.

(C) The American Society for Clinical Investigation, Inc. 0021-9738/95/04/1464/08 $\$ 2.00$

Volume 95, April 1995, 1464-1471 been observed in man and in laboratory animals in a variety of conditions, including fasting $(3,4)$, constant glucose infusion (5-8), continuous enteral nutrition $(9,10)$, meal ingestion (11-13), and oral glucose loads (14). These oscillations are disrupted in impaired glucose tolerance (15), abdominal obesity (16), and non-insulin-dependent diabetes $(15,17,18)$, suggesting that they may be important for the maintenance of normal glucose homeostasis. While a number of studies (19-22) have addressed the issue of the physiological significance of more rapid, lower amplitude, 10-15-min pulsations which are superimposed on the ultradian insulin oscillations, the potential role of the ultradian pattern of human insulin secretion in controlling daytime and overnight blood glucose levels has not yet been addressed.

This study was therefore designed to test the hypothesis that oscillatory insulin delivery with an ultradian periodicity is more efficient in reducing blood glucose levels than continuous administration throughout the 24-h cycle. Endogenous insulin secretion was suppressed by somatostatin, a constant intravenous glucose infusion was administered, and exogenous insulin was infused either at a constant rate or in a sinusoidal pattern with a period of $120 \mathrm{~min}$. The effects of oscillatory versus constant insulin delivery on blood glucose levels were compared during wakefulness and polygraphically recorded sleep.

\section{Methods}

Subjects. Studies were performed in nine normal young men (ages $21.3 \pm 0.7 \mathrm{yr}$ ). All were non-obese (body mass index $23.5 \pm 0.6 \mathrm{~kg} / \mathrm{m}^{2}$ ) and had normal fasting glucose $(4.66 \pm 0.16 \mathrm{mmol} / \mathrm{liter})$ and insulin $(48.9 \pm 6.6 \mathrm{pmol} / \mathrm{liter})$ levels. None had a history of endocrine or psychiatric disorder. They were all nonsmokers who were not taking any drugs. Shift workers or subjects having traveled across time zones within 60 $\mathrm{d}$ before the study were excluded. Subjects with irregular life habits or sleep complaints were also excluded.

Experimental protocol. The studies were carried out in the Clinical Research Center of the University of Chicago after written informed consent had been obtained. The experimental protocol was approved by the Institutional Review Board.

Each subject participated in two studies in randomized order. They were admitted in the evening of the day preceding each study, and an intravenous line was placed in each forearm, one for repeated blood sampling and one for fluid administration. Sleep was polygraphically monitored, and saline was infused to simulate the experimental conditions on the following day.

Throughout the study, the subjects did not consume any calories by mouth but were free to drink water and diet decaffeinated beverages. At 0800 , an infusion of $20 \%$ dextrose was started at a constant rate of $6 \mathrm{mg} / \mathrm{kg}$ per min, and blood sampling at 15-min intervals was initiated. To suppress endogenous insulin secretion, a constant infusion of somatostatin (Bachem California, Torrance, CA) at a rate of $140 \mu \mathrm{g} / \mathrm{h}$ was administered, and an infusion of exogenous insulin (Humulin R; Eli Lilly \& Co., Indianapolis, IN) at a mean rate of $0.3 \mathrm{mU} / \mathrm{kg}$ per min was given. Blood sampling and intravenous glucose, somatostatin and insulin infusions were continued for $28 \mathrm{~h}$. The subjects remained recum- 
bent throughout the study. During the night, they slept in total darkness from 2300 to 0700 , and sleep was polygraphically monitored in all but one subject. To avoid disturbing sleep, nocturnal blood sampling was performed from an adjacent room as described previously (23). Naps were not allowed. The same amount of insulin was infused in both studies, but in one study the rate of infusion was kept constant, whereas in the other study the rate of infusion was varied as a sine wave, with a period of $120 \mathrm{~min}$ and an amplitude of $30 \%$ above and below the mean. Oscillatory insulin administration was performed with a computer-controlled volumetric infusion pump (Flo-Gard 8000; Travenol Laboratories, Deerfield, IL). Glucose and insulin were measured in duplicate on each blood sample. C-peptide levels were measured at 15min intervals during the first $4 \mathrm{~h}$ of the study and then at hourly intervals. Plasma glucose was measured with the glucose oxidase technique using a semiautomatic analyzer (2300STAT; Yellow Springs Instrument Company, Yellow Springs, $\mathrm{OH}$ ) with a coefficient of variation of $<2 \%$. Serum insulin concentrations were determined by a double antibody technique with a lower level of sensitivity of $20 \mathrm{pmol} / \mathrm{liter}$ and an average intra-assay coefficient of variation of $6 \%$ (24). Plasma C-peptide levels were assayed as described previously (25). The lower limit of the assay is $20 \mathrm{pmol} /$ liter, and the intra-assay coefficient of variation averaged $6 \%$. Plasma glucagon levels were measured with a double antibody technique as previously described (26) with a lower detection limit of $15 \mathrm{ng} /$ liter and an average intra-assay coefficient of variation below $6 \%$.

Determination of insulin secretory rates. In each 15 -min blood sampling interval, the insulin secretion rate (ISR) ${ }^{1}$ was mathematically derived from plasma $\mathrm{C}$-peptide levels using a two-compartment model for C-peptide disappearance kinetics (27). The kinetic parameters were obtained from published demographic data adjusted for sex, age, and body surface area $(28)$. The mean $( \pm$ SEM) parameter values were $4.95 \pm 0.0 \mathrm{~min}$ for the short half-life, $32.2 \pm 0.10 \mathrm{~min}$ for the long halflife, and $0.76 \pm 0.0$ for the fraction of decay associated with the short half-life. The volume of distribution averaged $4.18 \pm 0.07$ liter.

Sleep recording and analysis. The polygraphic sleep records were scored visually at 30-s intervals in stages Wake, I, II, III, IV, and rapid eye movement (REM) according to the criteria of Rechtschaffen and Kales (29). Sleep onset and morning awakening were defined, respectively, as the times of occurrence of the first and last 30-s intervals scored II, III, IV, or REM. The sleep period time (SPT) was defined as the interval separating sleep onset from final morning awakening. Sleep efficiency was calculated as (SPT - total duration of Wake)/ total time in bed. Sleep maintenance was calculated as (SPT - total duration of Wake)/SPT.

Analysis of the glucose, insulin, and ISR profiles. To quantify the long-term (e.g., diurnal) changes in glucose and insulin independently of the ultradian oscillations, a smooth best-fit curve was calculated for each individual profile using the robust, nonlinear regression procedure proposed by Cleveland (30) with a window of $6 \mathrm{~h}$. This fitting procedure was applied to the 24-h glucose and insulin profiles collected after the first $4 \mathrm{~h}$ of the study, i.e., when levels of glucose, insulin, and C-peptide had stabilized after the transient elevation normally associated with the initiation of a glucose infusion. The nadirs and acrophases were defined, respectively, as the times of occurrence of minima and maxima in the best-fit curve. The value of a nadir (acrophase) was defined as the level of the best-fit curve at the nadir (acrophase). The amplitude of the diurnal variation was defined as $50 \%$ of the difference between the value of the acrophase and the value of the preceding nadir and was expressed in absolute concentration units or as a percentage of the 24 $h$ mean level.

Before quantifying the ultradian oscillations, the individual glucose and insulin profiles were smoothed using a two-point moving average as in previous studies of oscillations of insulin secretion $(9,31)$. This procedure consists of replacing the value observed at time $t$ by the

1. Abbreviations used in this paper: GH, growth hormone; ISR, insulin secretion rate; REM, rapid eye movement; SPT, sleep period time. arithmetic mean of the values observed at time $t-\Delta$ and $t$, where $\Delta$ is the sampling interval, i.e., $15 \mathrm{~min}$. This procedure strongly dampens all fluctuations shorter than $30 \mathrm{~min}$, allowing a better visualization of slower oscillations at the expense of a modest reduction in their amplitude. It also reduces measurement error by a factor of $\sqrt{2}$. All further calculations were performed on the smoothed profiles. To characterize the short-term (e.g., ultradian) variations in glucose and insulin independently of the diurnal rhythmicity, spectral analysis was performed on each individual series using smoothed spectral estimators as described by Jenkins and Watts (32). A Tukey window with a width of 48 data points was used. Before spectral analysis, the data were detrended by subtracting the regression curve quantifying the diurnal variation.

Pulses in glucose and insulin levels were identified using Ultra, a computer program for pulse detection, with a threshold for pulse significance of two times the intra-assay coefficient of variation (33). This analysis was performed on the detrended profiles smoothed by the twopoint moving average so that the measurement errors were divided by $\sqrt{2}$. Peaks of glucose and insulin levels were considered significant if their respective increments and decrements exceeded $2.8 \%$ (i.e., $2 \times 2 \%$ $\div \sqrt{2}$ ) and $8.5 \%$ (i.e., $2 \times 6 \% \div \sqrt{2}$ ), respectively. For each significant pulse, the increment was defined as the difference between the level at the peak and the level at the preceding trough, and was expressed in absolute concentration units (i.e., absolute increment) or as a percentage of the level at the preceding trough (i.e., relative increment). Group statistics on pulse increments were based on medians, rather than means, because of the non-Gaussian nature of their distributions.

Statistical tests. All results are expressed as mean \pm SEM. Differences between the conditions of constant versus oscillatory insulin infusion were examined by standard paired $t$ tests.

\section{Results}

Patterns of glucose, insulin and glucagon levels, and insulin secretion rates during constant and oscillatory insulin infusion. In both studies, after the initiation of intravenous somatostatin infusion, endogenous insulin secretion was rapidly suppressed from $85.5 \pm 8.2$ to $7.8 \pm 2.5 \mathrm{pmol} / \mathrm{min}$ within $25 \pm 2 \mathrm{~min}$. As is evident from the mean profiles shown in Fig. 1, the first few hours of glucose infusion were associated with a transient marked increase in plasma glucose levels, which resulted in a modest escape of endogenous insulin secretion from somatostatin suppression which was similar under both constant and oscillatory insulin infusion. Basal plasma glucagon levels were not different before the two studies $(80.9 \pm 8.2$ [constant] vs $78.6 \pm 5.1 \mathrm{ng} /$ liter [oscillatory]; $P=0.65$ ) and decreased similarly by an average of $45 \%$ during both studies $(45.9 \pm 5.3$ vs $41.1 \pm 3.7 \mathrm{ng} / \mathrm{liter} ; P=0.16)$. Exogenous insulin infusion acutely elevated serum insulin levels from $48.9 \pm 6.6 \mathrm{pmol} / \mathrm{liter}$ at 0800 to overall mean levels averaging $160.2 \pm 7.2 \mathrm{pmol} /$ liter during constant infusion and $153.2 \pm 6.3 \mathrm{pmol} /$ liter during oscillatory infusion $(P=0.05)$.

The upper panels of Fig. 1 illustrate the regular oscillations of serum insulin levels resulting from administration of exogenous insulin following a sinusoidal waveshape with a period of 120 min. Computer control of the infusion pump resulted in a highly reproducible profile of serum insulin and inter-individual synchronization of the oscillations. Well-defined oscillations in glucose levels followed each insulin oscillation throughout the study period. Representative serum insulin and plasma glucose profiles during the last $24 \mathrm{~h}$ of the study, i.e., after the transient period of equilibration, are shown for two individual subjects in Fig. 2. These profiles exemplify the higher efficiency of oscillatory versus constant insulin administration in controlling glucose levels. In subject 3, daytime glucose levels during the oscillatory condi- 

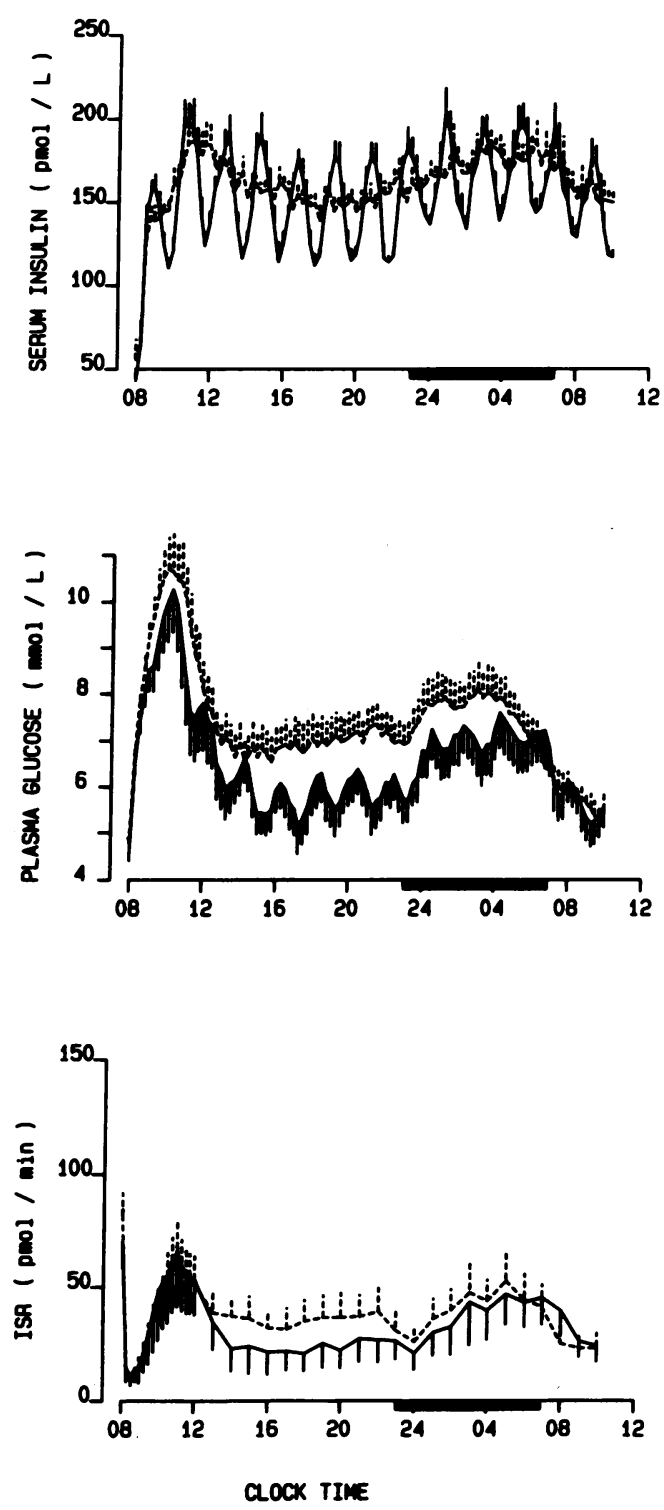

Figure 1. Mean profiles of serum insulin, plasma glucose, and ISR for the nine subjects receiving exogenous insulin either at a constant rate (broken line) or in an oscillatory pattern (solid line). The vertical bars represent the SEM. The horizontal black bars represent the scheduled sleep period.

tion were $3.23 \mathrm{mmol} /$ liter lower than during constant insulin administration. In subject 10 , the difference between the two conditions was smaller, but equally consistent, and averaged 1.53 mmol/liter between 1400 and the end of the study.

The mean glucose level over the entire study period was, on average, $0.72 \pm 0.31 \mathrm{mmol} /$ liter lower when insulin was infused in an ultradian oscillatory pattern than when the rate of infusion was kept constant $(P<0.05)$. Mean levels of serum insulin, plasma glucose, and ISR during the transient period, the daytime period, the sleep period, and the morning period are reported for both experimental conditions in Fig. 3. The greater hypoglycemic effect of oscillatory versus constant insulin infusion was particularly marked during the extended daytime period after the transient phase of glucose equilibration. Indeed, daytime blood glucose levels during the oscillatory con- dition were lower than during the constant condition in eight of the nine subjects, with the difference averaging $1.04 \pm 0.38$ mmol/liter $(n=9, P<0.03)$. No subject experienced any symptoms of hypoglycemia during the experiments. Oscillatory insulin infusion remained more efficient than constant administration throughout the first half of the sleep period. In the later part of the night and in the morning, the difference between the two modes of administration was no longer apparent. Remarkably, the lower daytime glucose concentrations during oscillatory insulin infusion were achieved in the face of levels of serum insulin and endogenous insulin secretion which tended to be lower than during constant infusion, although the same amount of exogenous insulin was administered under both conditions.

Quantitative characteristics of glucose and insulin oscillations. The quantitative characteristics of the ultradian oscillations in plasma glucose and serum insulin levels under both experimental conditions are summarized in Table I. Significant pulses in plasma glucose and serum insulin were detected even during constant glucose and insulin administration. In the absence of exogenous insulin oscillations, glucose pulses were more frequent than insulin pulses. In the oscillatory condition, the frequency and the magnitude of glucose and insulin pulses were significantly increased, and the ultradian patterns of peripheral concentrations closely matched those of the exogenous insulin infusion. The fact that the number of glucose pulses $(9.8 \pm 0.5$ per $24 \mathrm{~h})$ was less than the number of oscillations achieved in the exogenous infusion (i.e., 12 per $24 \mathrm{~h}$ ) reflects primarily the inability of the pulse detection program to identify oscillations with an incomplete upward or downward portion at the beginning or the end of the study period. The magnitude of the oscillations in serum insulin, as quantified by the relative pulse increment, averaged $\sim 60 \%$, indicating that the sinusoidal waveshape of the exogenous infusion (which had an amplitude of $30 \%$ around the mean level) was transmitted without significant dampening to peripheral concentrations. In contrast, the amplitude of the entrained glucose oscillations averaged $<20 \%$. Fig. 4 shows the mean spectra of the detrended insulin and glucose profiles under both experimental conditions. Under the condition of constant insulin and glucose infusion, spectral analysis confirmed the existence of inconsistent, low amplitude oscillations in the ultradian range (i.e., periods of 60-180 $\mathrm{min}$ ) in seven out of nine glucose profiles (peak period: $129 \pm 9 \mathrm{~min}$ ) and in all nine insulin profiles (peak period: $122 \pm 10 \mathrm{~min}$ ). Lower frequency variations tended to be more prominent for glucose than for insulin. Oscillatory insulin infusion had the effect of concentrating the spectral power in a narrow range around the exogenous period, i.e., $120 \mathrm{~min}$. Spectral power at 120 min was significantly higher for insulin than for glucose $(P$ $<0.0001$ ), indicating that the exogenous oscillation obliterated more endogenous variability for insulin than for glucose.

Diurnal variations of glucose and insulin levels. Despite the fact that the mean rates of infusion of insulin and glucose were constant throughout the study, robust diurnal variations of plasma glucose and serum insulin levels were observed in all subjects in both studies. A consistent pattern with an acrophase around midsleep and declining levels after morning awakening was identified by regression analysis. Significant nocturnal acrophases were observed in all but one subject for glucose and in all subjects for insulin. Individual examples are shown in Fig. 2. Because of the excellent inter-individual reproducibility, this diurnal rhythmicity is clearly apparent in the mean profiles 

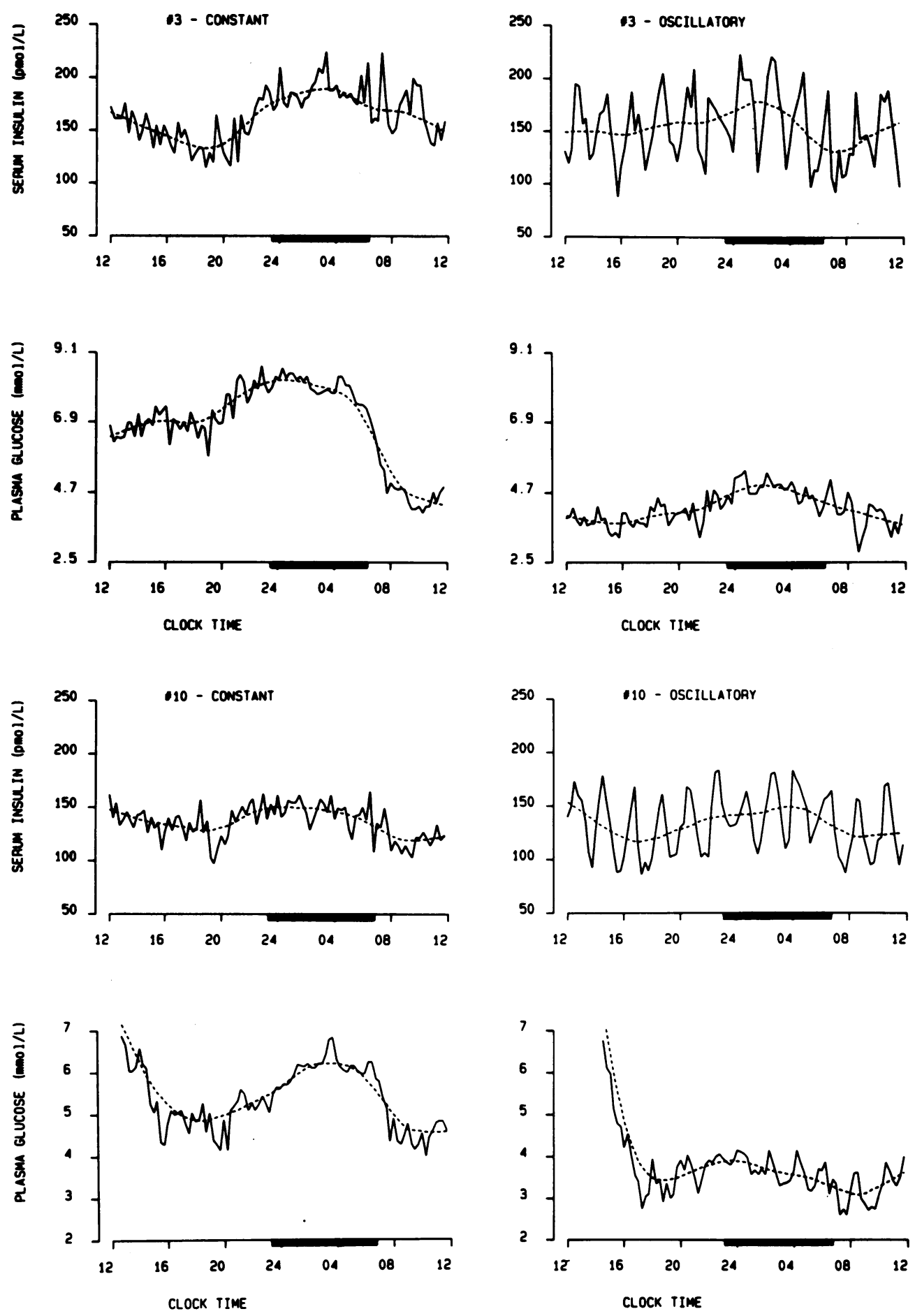

Figure 2. Individual profiles of serum insulin (top panels) and plasma glucose (lower panels) in two representative subjects receiving exogenous insulin either at a constant rate (left) or in an oscillatory pattern (right). Results are shown over the 24-h period after the initial 4-h transient period. The horizontal black bars represent the individual sleep periods as defined by polygraphic recordings. The regression curves are shown in dashed lines.

shown in Fig. 1. These diurnal variations in glucose and insulin levels were qualitatively and quantitatively similar during both constant and oscillatory insulin infusions.

Sleep onset and morning awakening were associated with robust changes in plasma glucose which were not readily apparent in the insulin profiles. In the majority of glucose profiles, well defined increases occurred immediately after sleep onset, and morning awakening was followed by a sharp decline (Figs. 1 and 2). All parameters quantifying sleep were similar under both conditions ( sleep efficiency: $76.0 \pm 13.3 \%$ vs $83.9 \pm 7.8 \%$; sleep maintenance: $89.6 \pm 8.1 \%$ vs $88.1 \pm 7.1 \%$; duration of stages III + IV: $93 \pm 31 \mathrm{~min}$ vs $92 \pm 21 \mathrm{~min}$; duration of REM stages; $68 \pm 24$ min vs $78 \pm 20 \mathrm{~min}$, during constant or oscillatory insulin infusion, respectively), except for the sleep period time which was longer under oscillatory $(458 \pm 20 \mathrm{~min})$ than constant $(408 \pm 59 \mathrm{~min})$ infusion.

\section{Discussion}

The present data demonstrate that insulin delivery in an oscillatory pattern with an ultradian period of $120 \mathrm{~min}$ is more efficient 


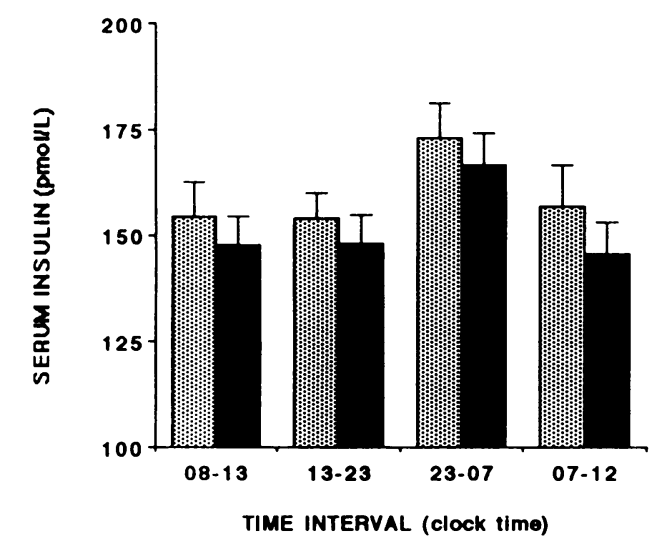

Table I. Quantitative Characteristics of the Ultradian Oscillations in Glucose and Insulin Levels during Constant and Oscillatory Insulin Infusion (Mean $\pm S E M ; n=9$ )

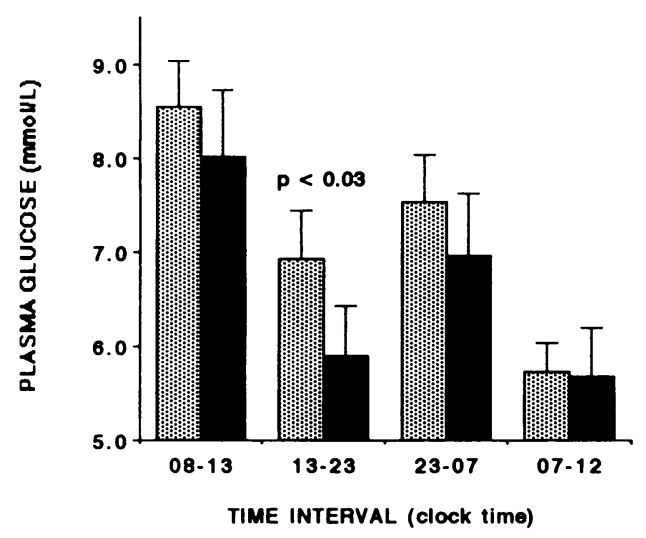

\begin{tabular}{|c|c|c|c|}
\hline & $\begin{array}{l}\text { Constant } \\
\text { infusion }\end{array}$ & $\begin{array}{c}\text { Oscillatory } \\
\text { infusion }\end{array}$ & \\
\hline \multicolumn{4}{|l|}{ Glucose } \\
\hline $\begin{array}{c}\text { Number of significan } \\
\text { pulses per } 24 \mathrm{~h} \\
\text { Median pulse increm }\end{array}$ & $7.6 \pm 0.5$ & $9.8 \pm 0.5$ & $P<0.02$ \\
\hline Absolute & $\begin{array}{l}0.83 \pm 0.06 \\
\mathrm{mmol} / \mathrm{liter}\end{array}$ & $\begin{array}{l}0.99 \pm 0.08 \\
\mathrm{mmol} / \mathrm{liter}\end{array}$ & $P<0.05$ \\
\hline Relative & $13.2 \pm 1.2 \%$ & $17.8 \pm 1.1 \%$ & $P<0.02$ \\
\hline \multicolumn{4}{|l|}{ Insulin } \\
\hline $\begin{array}{l}\text { Number of significan } \\
\text { pulses per } 24 \mathrm{~h}\end{array}$ & $3.6 \pm 0.5$ & $11.6 \pm 0.2$ & $P<0.0001$ \\
\hline Absolute & $\begin{array}{l}40.8 \pm 2.7 \\
\mathrm{pmol} / \text { liter }\end{array}$ & $\begin{array}{l}70.8 \pm 3.3 \\
\mathrm{pmol} / \text { liter }\end{array}$ & $P<0.0001$ \\
\hline Relative & $28.7 \pm 1.6 \%$ & $58.7 \pm 2.8 \%$ & $P<0.0001$ \\
\hline
\end{tabular}

suggest that the disruption of the ultradian oscillatory pattern of insulin secretion may play a role in the pathogenesis of abnormal glucose regulation and contribute to the development of insulin resistance.

The more marked hypoglycemic effect observed during oscillatory insulin infusion could theoretically result from a more pronounced inhibition of hepatic glucose output, an enhanced peripheral glucose disposal, or both. In response to the glucose and insulin concentrations prevailing in the present protocol, hepatic glucose output would be expected to be totally suppressed under both constant and oscillatory insulin infusion. Secretion of glucagon, the most potent stimulator of hepatic glucose production, was partially inhibited by the continuous infusion of somatostatin, and exogenous insulin administration resulted in an almost threefold increase in serum insulin levels throughout the study period. This moderate hyperinsulinemia was associated with elevated plasma glucose levels due to the exogenous glucose infusion, a combination that has been shown to strongly potentiate the suppression of hepatic glucose production (34). For these reasons, we believe that the greater hypoglycemic effect of oscillatory, rather than constant, insulin infusion is due to an effect on peripheral glucose utilization rather than hepatic glucose production. An earlier study performed in dogs showed the existence of ultradian glucose oscillations despite almost complete suppression of endogenous glucose production, demonstrating a causal relationship to variations in peripheral glucose uptake (6). In a subsequent study using the hyperglycemic clamp technique, evidence that glucose oscillations enhance the efficiency and stability of glucose disposal was obtained (35).

The complex temporal organization of human insulin secretion also includes more rapid (i.e., $10-15 \mathrm{~min}$ ) pulsations that are superimposed on the ultradian oscillations $(3,36-38)$. A number of studies have examined the possible physiological significance of the rapid insulin pulses by comparing intermittent versus continuous insulin infusion in normal and/or diabetic subjects. Matthews et al. first demonstrated (19) in normal subjects a significant reduction in plasma glucose concentrations 

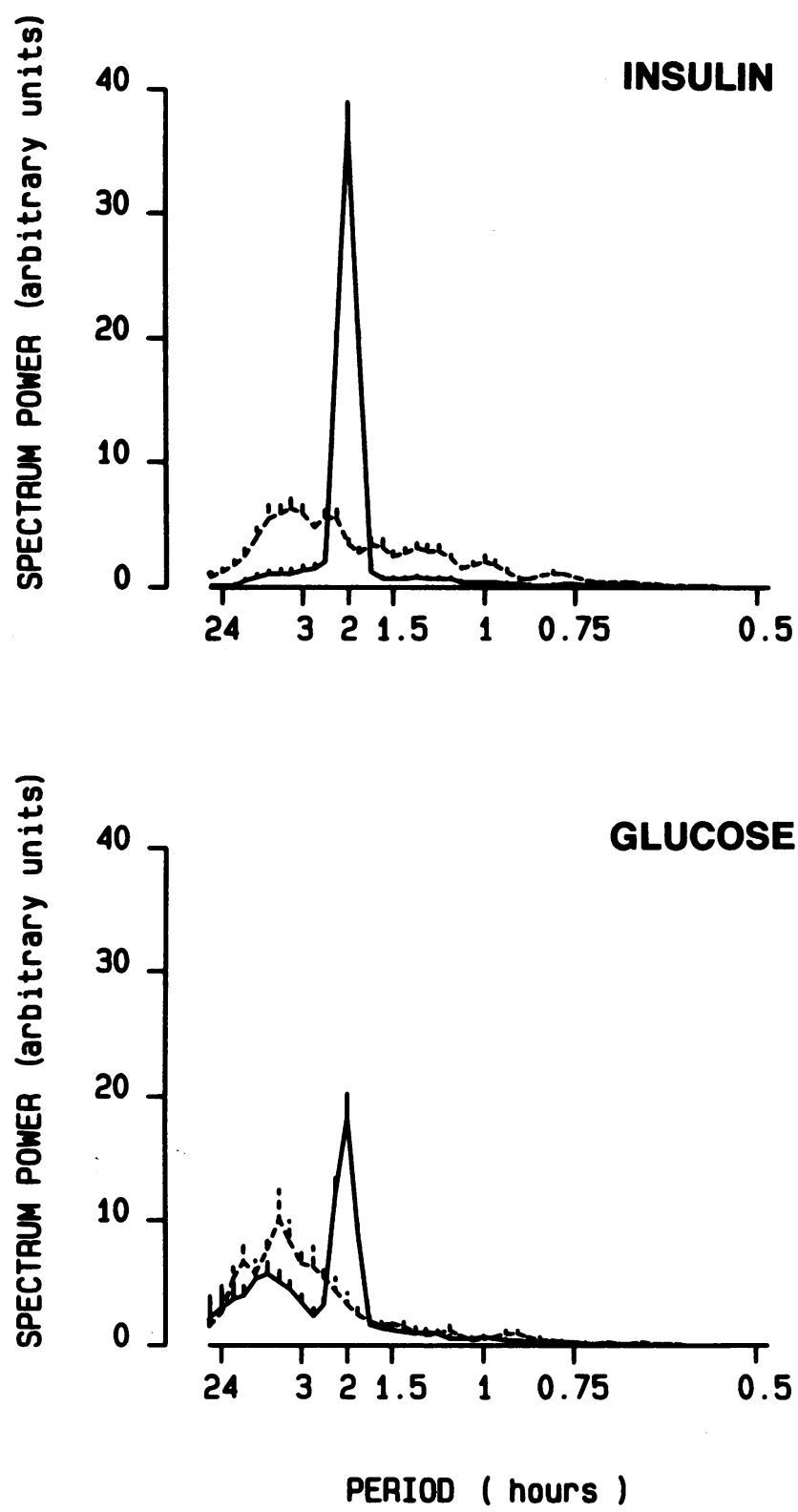

Figure 4. Mean spectral estimations for detrended serum insulin (top) and plasma glucose (bottom) profiles in subjects studied under exogenous insulin at a constant rate (broken lines) or in an oscillatory pattern (solid line). Error bars represent the SEM. Each individual spectrum was normalized assuming that the total variance of the series is $100 \%$ in order to obtain meaningful comparisons.

during pulsatile, as compared with constant, insulin infusion with a maximum difference of $0.87 \mathrm{mmol} /$ liter achieved only after 8-9 h of insulin administration. Further studies (20-22) combining measurements of glucose kinetics and the glucose clamp technique showed that, in contrast to the mechanism underlying the efficacy of the ultradian oscillations, the greater hypoglycemic effect of the rapid pulses is entirely due to a more marked inhibition of hepatic glucose production, without involvement of peripheral glucose utilization. The two oscillatory periodicities of insulin thus appear to each represent an optimal functional status of a different critical component of the insulin-glucose regulation.
The concept that the rapid pulses act on glucose production while the ultradian oscillations have an effect on peripheral glucose utilization is also supported by other findings. The rapid insulin pulses are large in the portal vein, immediately before passing through the liver (39). However, because of their short periodicity, they are much smaller in the peripheral circulation and would be even smaller in the interstitium, from where insulin acts peripherally. The amplitude of the signal emanating from the rapid pulses that could be involved in regulating glucose utilization is therefore necessarily very small, and it is thus expected that effects on glucose utilization would be minimal. In contrast, the ultradian oscillations are quite large in the peripheral blood circulation, particularly when stimulated by intravenous glucose, during continuous enteral nutrition, and after meals $(4,9,12,13)$, physiological states when glucose utilization is high. Additionally, because of the longer periodicity, the ultradian signal can be transmitted to the interstitium at a significant amplitude, permitting an effect on glucose utilization.

The molecular mechanisms underlying the greater efficacy of insulin delivery in an ultradian oscillatory mode, rather than at a constant rate, need to be elucidated. Recent findings suggest that this greater efficacy could be due to enhanced insulin receptor binding. Indeed, fluctuating changes in free-ligand concentration have been shown to enhance the exchange of ligand previously bound to the insulin growth factor-I (IGF-I) receptor, a heterotetramer structure similar to the insulin receptor, suggesting that oscillating hormonal levels may lead to overall enhanced function (40). This effect was attributed to the fact that each receptor is capable of binding two molecules, but under conditions of stable ligand concentrations very few receptors, if any, are doubly occupied. In contrast, when the ligand concentration increases, a transient elevation in doubly bound receptors occurs, leading to an increased exchange of receptorbound molecules (40). Under laboratory conditions, when IGFI receptors are exposed to an oscillatory rather than constant IGF-I concentration, an increased overall IGF-I receptor binding is observed (Tager, H. S., personal communication). Because of the structural similarity between the IGF-I and insulin receptors, analogous results should be expected for the binding and exchange of insulin. The greater hypoglycemic effect of oscillatory, rather than constant, insulin delivery could thus be mediated by increased binding and/or exchange of insulin to its receptor.

Ultradian oscillations in plasma glucose persisted even when insulin was infused at a constant rate. This finding raises the possibility that factors other than oscillations in insulin may be involved in the generation of ultradian glucose oscillations.

Irrespective of the mode of insulin infusion, a large and highly reproducible diurnal variation in the set-point of glucose regulation, with higher levels of glucose and insulin during the evening and sleep periods, was apparent despite the continuous infusion of somatostatin and glucose at a constant rate. We have previously observed a similar diurnal variation during prolonged constant glucose infusion without suppression of endogenous insulin secretion $(7,8)$ and demonstrated that it reflects the combined effects of circadian rhythmicity and sleep, two important and independent physiological modulators of glucose regulation (23). Glucose increases after sleep onset were found to correlate with nocturnal growth hormone (GH) secretion (23). The persistence of a robust diurnal variation in glucose and insulin levels in the absence of endogenous insulin secretion 
and in the face of glucose and insulin infusions at constant mean rates confirms the findings of previous studies showing that reduced insulin sensitivity later in the day is a major cause of the reduction in glucose tolerance $(41,42)$. Involvement of GH secretion in causing the additional hyperglycemic effect of sleep may not be excluded because the dose of somatostatin used in this study was probably insufficient to suppress nocturnal GH secretion.

In this study, as well as in previous investigations $(7,8$, 23 ), the pattern of plasma glucose changes observed during the night was different from that described as the "dawn phenomenon" in diabetic patients (43) and, in some studies but not in others, in nondiabetic subjects $(44,45)$. Indeed, we observed a late evening/early nocturnal glucose rise followed by a late nocturnal glucose fall instead of the early morning glucose increase referred to as the dawn phenomenon. An important difference between the experimental conditions used here and those under which the dawn phenomenon has been observed is that a fasting state was not established since our subjects received a constant rate of glucose infusion. Thus, the stimulation of nocturnal GH secretion, which has been implicated in the pathogenesis of the dawn phenomenon during an overnight fast (46), did not occur in our study.

In conclusion, in normal subjects, ultradian oscillations in insulin secretion appear to promote more efficient glucose utilization, thereby increasing the hypoglycemic effect of insulin. These findings raise the possibility that disturbances in the normal ultradian pattern of insulin secretion may aggravate the insulin resistance frequently present in subjects with impaired glucose tolerance or non-insulin-dependent diabetes mellitus. The hypothesis that the restoration of an ultradian pattern of insulin oscillations in conditions of glucose intolerance may contribute to the normalization of glucose control should therefore be tested.

\section{Acknowledgments}

We thank the nursing staff of the General Clinical Research Center of the University of Chicago for skillful assistance and Ms. S. Santos for expert completion of sleep recordings and scoring. Mr. P. Rue, Ms. M. Jackson, and Mr. W. Pugh are acknowledged for their technical assistance. We are indebted to the late Dr. Howard S. Tager for valuable discussions about the interaction of insulin and IGF-I with their receptors.

These studies were supported by grants DK-41814 and DK-31842 from the National Institutes of Health to E. Van Cauter and K. S. Polonsky, NATO Scientific Exchange Programme Award 910653 (Patterns of Insulin Secretion and Underlying Nonlinear Dynamic Models), grant DK-26678 to the Diabetes Research and Training Center of the University of Chicago, and by grant RR-00055 to the General Clinical Research Center of the University of Chicago. Dr. Sturis is the recipient of a Research Career Development Award from the Juvenile Diabetes Foundation International.

\section{References}

1. Belchetz, P. E., T. M. Plant, Y. Nakai, E. J. Keogh, and E. Knobil. 1978. Hypophysial responses to continuous and intermittent delivery of hypothalamic gonadotropin releasing hormone. Science (Wash. DC). 202:631-633.

2. Hastings, M. H. 1991. Neuroendocrine rhythms. Pharmacol. \& Ther. 50:3571.

3. Sturis, J., K. S. Polonsky, E. T. Shapiro, J. D. Blackman, N. M. O’Meara, and $\mathrm{E}$. Van Cauter 1992. Abnormalities in the ultradian oscillations of insulin secretion and glucose levels in type 2 (non-insulin-dependent) diabetic patients. Diabetologia. 35:681-689.
4. Sturis, J., N. M. O'Meara, E. T. Shapiro, J. D. Blackman, H. Tillil, K. S. Polonsky, and E. Van Cauter. 1993. Differential effects of glucose stimulation upon rapid pulses and ultradian oscillations of insulin secretion. J. Clin. Endocrinol. \& Metab. 76:895-901.

5. Ookhtens, M., D. J. Marsh, S. W. Smith, R. N. Bergman, and F. E. Yates 1974. Fluctuations of plasma glucose and insulin in conscious dogs receiving glucose infusions. Am. J. Physiol. 226:910-919.

6. Bowden, C. R., R. N. Bergman, and D. J. Marsh. 1980. Cause of glucose oscillations during glucose infusion: periodic variation in glucose uptake. Am. J. Physiol. 238:E395-E407.

7. Shapiro, E. T., H. Tillil, K. S. Polonsky, V. S. Fang, A. H. Rubenstein, and $\mathrm{E}$. Van Cauter. 1988. Oscillations in insulin secretion during constant glucose infusion in normal man: relationship to changes in plasma glucose. J. Clin. Endocrinol. \& Metab. 67:307-314.

8. Van Cauter, E., D. Désir, C. Decoster, F. Féry, and E. O. Balasse. 1989. Nocturnal decrease in glucose tolerance during constant glucose infusion. J. Clin. Endocrinol. \& Metab. 69:604-611.

9. Simon, C., G. Brandenberger, and M. Follenius. 1987. Ultradian oscillations of plasma glucose, insulin, and C-peptide in man during continuous enteral nutrition. J. Clin. Endocrinol. \& Metab. 64:669-674.

10. Sonnenberg, G. E., R. G. Hoffmann, C. P. Johnson, and A. H. Kissebah 1992. Low- and high-frequency insulin secretion pulses in normal subjects and pancreas transplant recipients. Role of extrinsic innervation. J. Clin. Invest. 90:545-553.

11. Robbins, D. C., J. Jaspan, B. Vasquez, and E. Van Cauter. 1987. Biphasic patterns of peripheral insulin and glucose levels after lunch in normal subjects. Diabetes Care. 10:293-299.

12. Simon, C., M. Follenius, and G. Brandenberger. 1987. Postprandial oscillations of plasma glucose, insulin and C-peptide in man. Diabetologia. 30:769773.

13. Polonsky, K. S., B. D. Given, and E. Van Cauter. 1988. 24-Hour profiles and pulsatile patterns of insulin secretion in normal and obese subjects. J. Clin. Invest. 81:442-448.

14. Kraegen, E. W., J. D. Young, E. P. George, and L. Lazarus. 1972. Oscillations in blood glucose and insulin after oral glucose. Horm. Metab. Res. 4:409413.

15. O'Meara, N. M., J. Sturis, E. Van Cauter, and K. S. Polonsky. 1993. Lack of control by glucose of ultradian insulin secretory oscillations in impaired glucose tolerance and in non-insulin-dependent diabetes mellitus. J. Clin. Invest. 92:262271

16. Sonnenberg, G. E., R. G. Hoffman, R. A. Mueller, and A. H. Kissebah. 1994. Splanchnic insulin dynamics and secretion pulsatilities in abdominal obesity. Diabetes. 43:468-477.

17. Polonsky, K. S., B. D. Given, L. J. Hirsch, H. Tillil, E. T. Shapiro, C. Beebe, B. H. Frank, J. A. Galloway, and E. Van Cauter. 1988. Abnormal patterns of insulin secretion in non-insulin-dependent diabetes mellitus. N. Engl. J. Med. 318:1231-1239.

18. Simon, C., G. Brandenberger, M. Follenius, and J. L. Schlienger. 1991. Alteration in the temporal organisation of insulin secretion in type 2 (non-insulindependent) diabetic patients under continuous enteral nutrition. Diabetologia. 34:435-440.

19. Matthews, D. R., B. A. Naylor, R. G. Jones, G. M. Ward, and R. C Turner. 1983. Pulsatile insulin has greater hypoglycemic effect than continuous delivery. Diabetes. 32:617-621.

20. Verdin, E., M. Castillo, A. S. Luyckx, and P. J. Lefebvre. 1984. Similar metabolic effects of pulsatile versus continuous human insulin delivery during euglycemic, hyperinsulinemic glucose clamp in normal man. Diabetes. 33:11691174.

21. Bratusch-Marrain, P. R., M. Komjati, and W. K. Waldhausl. 1986. Efficacy of pulsatile versus continuous insulin administration on hepatic glucose production and glucose utilization in type I diabetic humans. Diabetes. 35:922-926.

22. Paolisso, G., A. J. Scheen, D. Giugliano, S. Sgambàto, A. Albert, M. Varricchio, F. D'Onofrio, and P. J. Lefebvre. 1991. Pulsatile insulin delivery has greater metabolic effects than continuous hormone administration in man importance of pulse frequency. J. Clin. Endocrinol. \& Metab. 72:607-615.

23. Van Cauter, E., J. D. Blackman, D. Roland, J. P. Spire, S. Refetoff, and K. S. Polonsky. 1991. Modulation of glucose regulation and insulin secretion by circadian rhythmicity and sleep. J. Clin. Invest. 88:934-942.

24. Morgan, C. R., and A. Lazarow. 1963. Immunoassay of insulin: two antibody system: plasma insulin levels of normal, subdiabetic and diabetic rats. Diabetes. 12:115-126.

25. Faber, O. K., C. Binder, J. Markussen, L. G. Heding, V. K. Naithani, H. Kuzuya, P. Blix, D. L. Horwitz, and A. H. Rubenstein. 1978. Characterization of seven C-peptide antisera. Diabetes. 27(Suppl. 1):170-177.

26. Soybel, D., J. Jaspan, K. Polonsky, I. Goldberg, E. Rayfield, and H. Tager. 1983. Differential immunoreactivity of plasma glucagon antibodies. J. Clin. Endo crinol. \& Metab. 56:612-618.

27. Polonsky, K. S., J. Licinio-Paixao, B. D. Given, W. Pugh, P. Rue, J. Galloway, T. Karrison, and B. Frank. 1986. Use of biosynthetic human C-peptide 
in the measurement of insulin secretion rates in normal volunteers and type $I$ diabetic patients. J. Clin. Invest. 77:98-105.

28. Van Cauter, E., F. Mestrez, J. Sturis, and K. S. Polonsky. 1992. Estimation of insulin secretion rates from C-peptide levels: comparison of individual and standard kinetic parameters for C-peptide clearance. Diabetes. 41:368-377.

29. Rechtschaffen, A., and A. Kales. 1968. A Manual of Standardized Terminology, Techniques and Scoring System for Sleep Stages of Human Subjects. Government Printing Office, Washington, DC.

30. Cleveland, W. S. 1979. Robust locally weighted regression and smoothing scatterplots. J. Am. Stat. Assoc. 74:829-836.

31. Sturis, J., E. Van Cauter, J. D. Blackman, and K. S. Polonsky. 1991. Entrainment of pulsatile insulin secretion by oscillatory glucose infusion. J. Clin. Invest. 87:439-445.

32. Jenkins, G. M., and D. G. Watts. 1968. Spectral Analysis and Its Application. Holden Day, San Francisco. 525 pp.

33. Van Cauter, E. 1988. Estimating false-positive and false-negative error in analyses of hormonal pulsatility. Am. J. Physiol. 254:E786-E794.

34. DeFronzo, R. A., and E. Ferrannini. 1987. Regulation of hepatic glucose metabolism in humans. Diabetes Metab. Rev. 3:415-459.

35. Marsh, B. D., D. J. Marsh, and R. N. Bergman. 1986. Oscillations enhance the efficiency and stability of glucose disposal. Am. J. Physiol. 250:E576-E582

36. Lang, D. A., D. R. Matthews, J. Peto, and R. C. Turner. 1979. Cyclic oscillations of basal plasma glucose and insulin concentrations in human beings N. Engl. J. Med. 301:1023-1027.

37. Hansen, B. C., K. C. Jen, S. B. Pek, and R. A. Wolfe. 1982. Rapid oscillations in plasma insulin, glucagon, and glucose in obese and normal weigh humans. J. Clin. Endocrinol. \& Metab. 54:785-792.
38. Matthews, D. R., D. A. Lang, M. A. Burnett, and R. C. Turner. 1983. Control of pulsatile insulin secretion in man. Diabetologia. 24:231-237.

39. Jaspan, J. B., E. Lever, K. S. Polonsky, and E. Van Cauter. 1986. In vivo pulsatility of pancreatic islet peptides. Am. J. Physiol. 251:E215-E226.

40. Zhong, P., J. F. Cara, and H. S. Tager. 1993. Importance of receptor occupancy, concentration differences, and ligand exchange in the insulin-like growth factor I receptor system. Proc. Natl. Acad. Sci. USA. 90:11451-11455.

41. Lee, A., M. Ader, G. A. Bray, and R. N. Bergman. 1992. Diurnal variation in glucose tolerance: cyclic suppression of insulin action and insulin secretion in normal-weight, but not obese, subjects. Diabetes. 41:742-749.

42. Verrillo, A., A. De Teresa, C. Martino, G. Di Chiara, M. Pinto, L. Verrillo, F. Torello, and A. Gattoni. 1989. Differential roles of splanchnic and periphera tissues in determining diurnal fluctuation of glucose tolerance. Am. J. Physiol. 257:E459-E465.

43. Bolli, G. B., and J. E. Gerich. 1984. The "dawn phenomenon"- - a common occurrence in both non-insulin-dependent and insulin-dependent diabetes mellitus. N. Engl. J. Med. 310:746-750.

44. Bolli, G. B., P. De Feo, S. De Cosmo, G. Perriello, M. M. Ventura, F. Calcinaro, C. Lolli, P. Campbell, P. Brunetti, and J. E. Gerich. 1984. Demonstration of the dawn phenomenon in normal volunteers. Diabetes. 33:1150-1153.

45. Simon, C., M. Follenius, and G. Brandenberger. 1988. Absence of the dawn phenomenon in normal subjects. J. Clin. Endocrinol. \& Metab. 67:203205.

46. Campbell, P. J., G. B. Bolli, P. E. Cryer, and J. E. Gerich. 1985. Pathogenesis of the dawn phenomenon in patients with insulin-dependent diabetes mellitus : accelerated glucose production and impaired glucose utilization due to nocturnal surges in growth hormone secretion. N. Engl. J. Med. 312:1473-1479. 